\title{
Subdural fluid collection and hydrocephalus following cervical schwannoma resection: hydrocephalus resolution after spinal pseudomeningocele repair: case report
}

\author{
Nicola Benedetto, MD, PhD, Federico Cagnazzo, MD, Carlo Gambacciani, MD, and \\ Paolo Perrini, MD, PhD
}

Neurosurgical Department, Azienda Ospedaliero Universitaria Pisana, Pisa, Italy

The authors report the case of a 31-year-old man who developed neck pain and headache 2 months after the uncomplicated resection of a cervical schwannoma. MR imaging revealed infratentorial subdural fluid collections and obstructive hydrocephalus associated with cervical pseudomeningocele. The clinical symptoms, subdural fluid collections, and ventricular dilation resolved after surgical correction of the pseudomeningocele. This report emphasizes that hydrocephalus may be related to disorders of cerebrospinal fluid flow dynamics induced by cervical pseudomeningocele. In these rare cases, both the hydrocephalus and the symptoms are resolved by the simple correction of the pseudomeningocele.

http://thejns.org/doi/abs/10.3171/2016.5.SPINE16153

KEY WORDS hydrocephalus; pseudomeningocele; spinal tumor; subdural fluid collection; cervical; oncology

$\mathrm{H}$ YDROCEPHALUS following spinal tumor resection is a rare occurrence. ${ }^{3,5,14}$ Hyperproteinorrachia, subarachnoid blood-contaminated CSF, and secondary basal arachnoiditis are considered to be the etiological factors. ${ }^{3}$ In some instances, the development of postoperative hydrocephalus may be associated with abrupt clinical deterioration requiring ventriculoperitoneal (VP) shunt placement to relieve increased intracranial pressure. The appearance of postoperative ventricular dilation and subdural fluid collections is extremely unusual and poses pathophysiological and therapeutic dilemmas. In this report, we illustrate a case of subacute hydrocephalus associated with subdural fluid collection and spinal pseudomeningocele occurring a few months after cervical schwannoma resection. The resolution of hydrocephalus following pseudomeningocele repair sparks new insights into the pathogenesis of this unusual condition.

\section{Case Report}

History and Examination

A 31-year-old man with a 2-year history of clumsiness in his left hand underwent an uneventful resection of a C4-6 dumbbell schwannoma at another hospital (Fig.
1). Two months later, he was admitted to our department with symptoms of neck pain and headache. The patient was afebrile and no CSF leakage was detected. MRI of the cervical spine revealed a significant pseudomeningocele extending from C-4 to C-6 (Fig. 2). MRI of the brain demonstrated infratentorial subdural fluid collections and a triventricular hydrocephalus (Evan's index 0.33) (Fig. 2).

\section{Operation}

The patient's hydrocephalus was considered to be related to subdural fluid collections and pseudomeningocele. It was thus decided to treat the pseudomeningocele first, with a VP shunt only being considered as a second option. The patient underwent pseudomeningocele exploration and dural repair. No lumbar drainage was inserted after induction of general anesthesia or postoperatively. Under microscopic magnification the dural breach was explored, which revealed intermittent CSF leaks during the systolic phase through a small arachnoid defect and protrusion of the spinal cord through the dural-arachnoid defect during the diastolic phase. The arachnoid veil protruding through the dural breach was widely fenestrated, and the dura was rendered watertight with an artificial graft by using 4-0 nonabsorbable sutures (Fig. 3). 


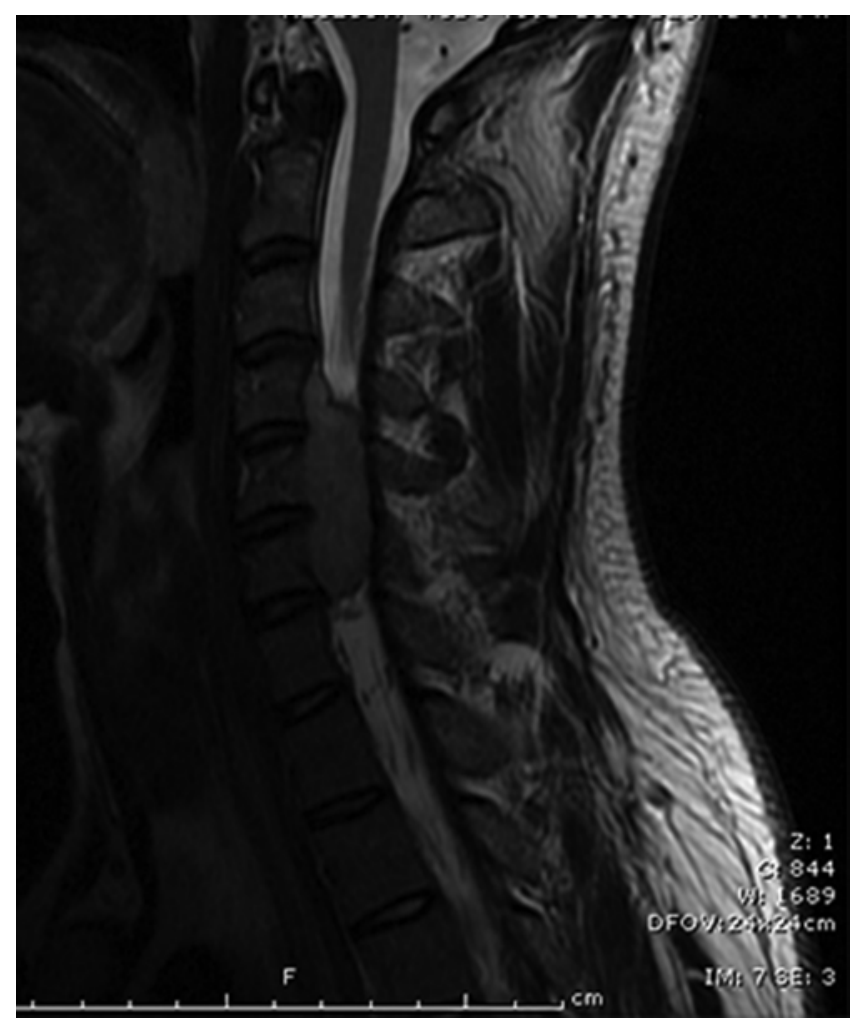

FIG. 1. Preoperative sagittal T2-weighted MR image showing a C4-6 spinal schwannoma severely compressing the spinal cord.

\section{Postoperative Course}

After the operation, the patient experienced immediate improvement in his preoperative symptoms, and a CT scan revealed that normal ventricular size (Evan's index 0.26 ) had been restored. At the 1-month follow-up, the patient was headache free, and an MRI study obtained at that time revealed that both the hydrocephalus and infratentorial subdural fluid collections had been resolved. Resolution of the cervical pseudomeningocele was also evident (Fig. 4).

\section{Discussion}

The association of hydrocephalus and intraspinal tumors is a rare but well-known condition, which usually resolves following tumor resection. ${ }^{9}$ Several hypotheses have been made to explain this association, including hyperproteinorrachia with CSF hyperviscosity, spinal subarachnoid hemorrhage, and epidural venous compression., 5,14 The occurrence of hydrocephalus after resection of intraspinal tumors is extremely unusual. Bland and $\mathrm{McDonald}^{3}$ reported the case of a 58-year-old man who developed communicating hydrocephalus 1 month after an otherwise uneventful resection of a cervical schwannoma. The patient presented with signs of intracranial hypertension and underwent VP shunt placement, resulting in resolution of symptoms. The authors speculated that both hyperproteinorrachia and subarachnoid bleeding might have been the etiological factors in the development of hydrocephalus.

Montemurro et al. ${ }^{10}$ described a case of hydrocephalus associated with craniocervical pseudomeningocele after
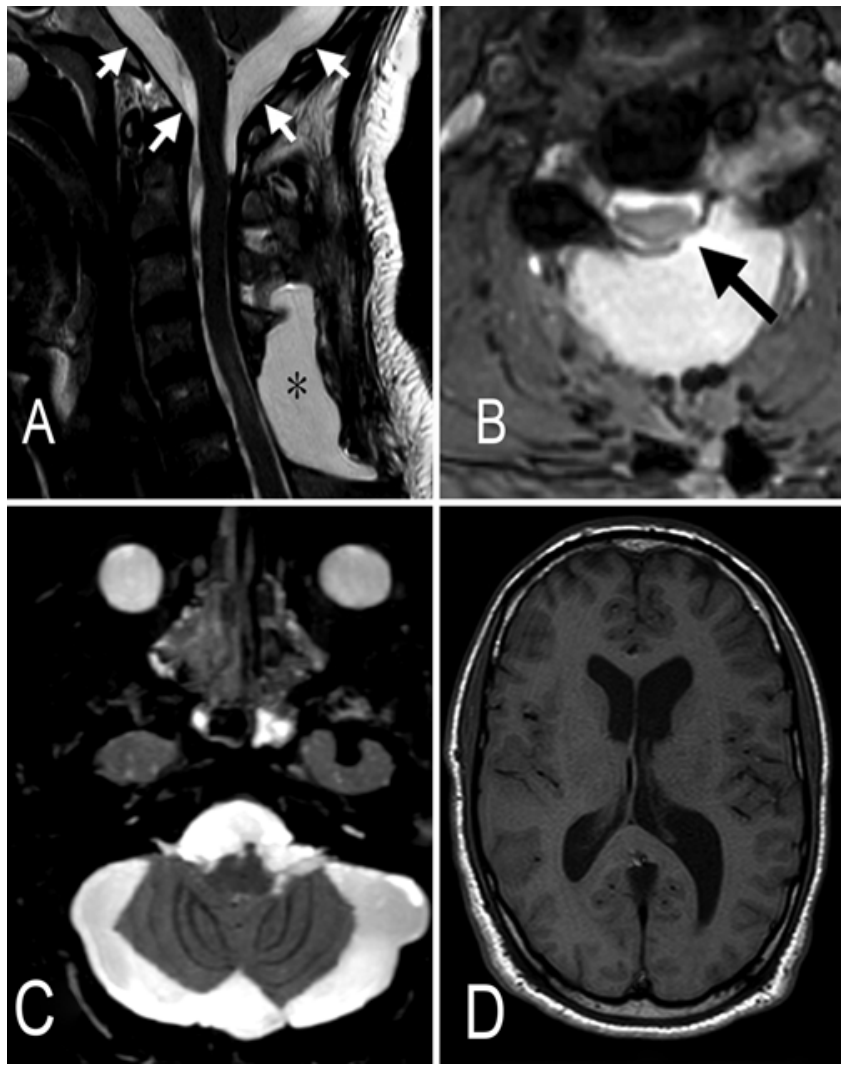

FIG. 2. MR images obtained 2 months after schwannoma resection. A: Sagittal T2-weighted MR image of the cervical spine, demonstrating a cervical pseudomeningocele (asterisk) and significant infratentorial subdural fluid collections extending to the craniovertebral junction (arrows). B: Axial T2-weighted MR image at the C-5 level, demonstrating the pseudomeningocele and the dural defect (arrow). C and D: Axial T2-weighted MR image obtained using the SENSE (sensitivity encoding) technique (C), and axial T1-weighted MR image of the brain revealing infratentorial subdural fluid collections and hydrocephalus (D).

the resection of a dumbbell-shaped C-2 neurofibroma in a patient with neurofibromatosis Type 1 . The authors treated the patient with VP shunting and hypothesized that postoperative pseudomeningocele at the craniocervical junction had interfered with the free CSF flow, resulting in progressive ventricular dilation.

Our case differs from the other reported cases in that ventricular dilation occurred in association with infratentorial subdural fluid collection and cervical pseudomeningocele. Although the present report shares some similari-


FIG. 3. Intraoperative photographs demonstrating the dural-arachnoid defect exposed after pseudomeningocele evacuation $(\mathbf{A})$ and its watertight closure (B). Figure is available in color online only. 

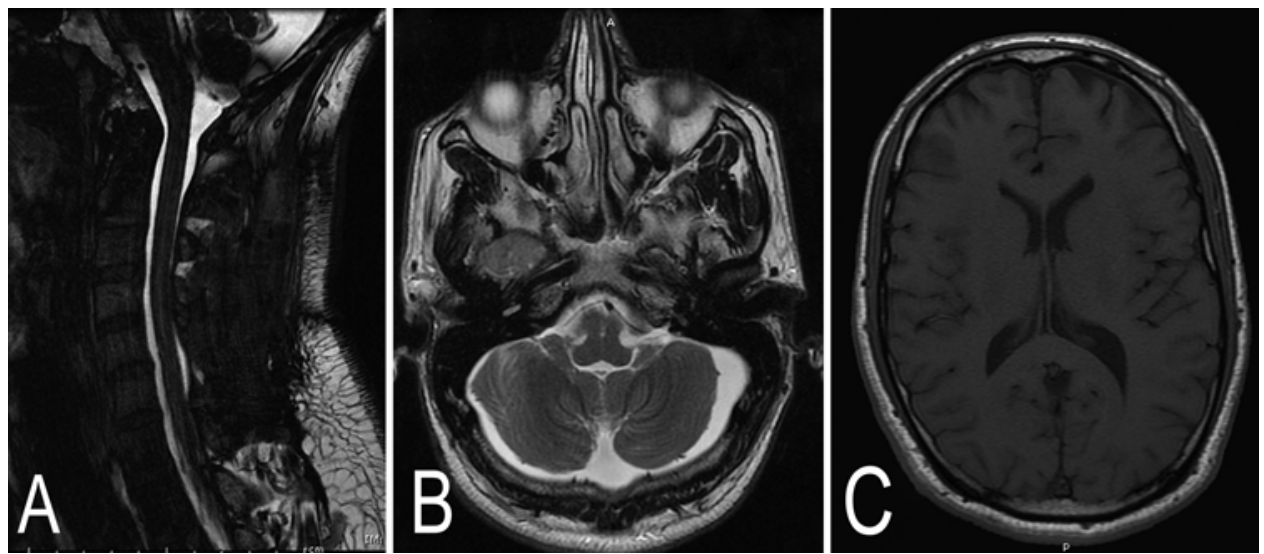

FIG. 4. MR images obtained 1 month after pseudomeningocele repair. A: Sagittal T2-weighted MR image of the cervical spine demonstrating pseudomeningocele resolution. B and C: Axial T2- and T1-weighted MR images of the brain showing sensible reduction of infratentorial subdural fluid collections and normalization of ventricular size.

ties with reported cases of external hydrocephalus, ${ }^{4,7,8,15}$ its pathophysiological mechanism is probably different. External hydrocephalus, which was originally described in pediatric patients with enlargement of the subarachnoid or subdural space in association with increased intracranial pressure, ${ }^{1}$ has been subsequently described in adults after trauma and subarachnoid hemorrhage. ${ }^{4,7}$ Paradoxically, in external hydrocephalus the ventricular system increases in size when the CSF accumulates in the subdural space..$^{2,4,15}$ Although the underlying mechanisms of this condition are largely unknown, it has been proposed that a tear in the arachnoid membrane and an imbalance between production and absorption of CSF play a pivotal role in its pathophysiology. ${ }^{4,15}$ In the present case, the development of infratentorial subdural fluid collection exerted compression of the cerebellum with resultant obstructive hydrocephalus. We propose that 2 key factors are relevant to our case: a dural-arachnoid tear, and the occurrence of cervical pseudomeningocele. It is conceivable that the small dural- arachnoid defect led to pseudomeningocele formation, acting as a ball-valve mechanism (Fig. 5). In this scenario, the defect allowed a 1-way flow of CSF toward the postoperative bed of the paraspinal cervical musculature. According to Laplace's law, the pressure in the pseudomeningocele remained relatively stable until the radius of the cavity continued to increase. When the paraspinal muscles hindered the cavity growth, the pressure inside the pseudomeningocele increased and the CSF dissected the spinal dura-arachnoid interface (Fig. 6). This concept was previously hypothesized by Ranjan and Cast..$^{13}$ In addition, the separation of arachnoid and dura eventually extended toward the subdural space of the craniovertebral junction and the posterior fossa. The development of subdural fluid collections in the posterior fossa led to compression of the cerebellum and the fourth ventricle and obstruction of CSF circulation in the posterior fossa cisterns with resultant triventricular hydrocephalus. Interestingly, the occurrence of hydrocephalus associated with subdural fluid collections



FIG. 5. Illustration demonstrating the proposed pathophysiology of pseudomeningocele collection. During systole, the CSF moves from the spinal subarachnoid space into the pseudomeningocele cavity and passes through the cervical dural-arachnoid defect, which acts as a ball-valve mechanism. In the diastolic phase, the lowered spinal subarachnoid pressure allows functional closure of the defect, preventing the return of CSF into the subarachnoid space. Figure is available in color online only. 


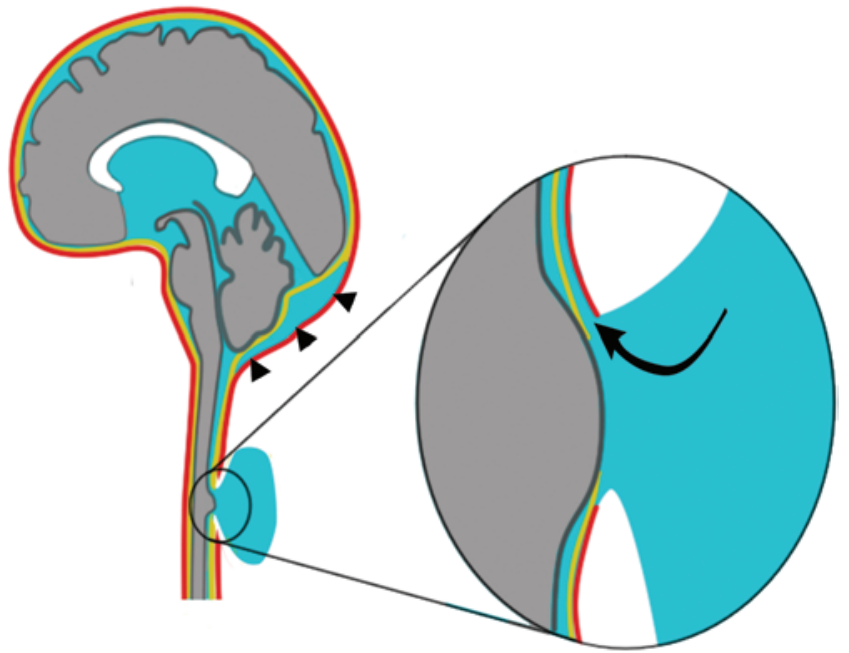

FIG. 6. Illustration demonstrating the proposed mechanism underlying the development of subdural fluid collection and obstructive hydrocephalus. When the pressure inside the pseudomeningocele is balanced with the wall tension, the trapped CSF dissects the dura-arachnoid interface layer, producing subdural fluid collections. The dissection eventually extends toward the craniovertebral junction and the posterior fossa where the subdural fluid collections compress the cerebellum and obstruct the free CSF flow, inducing obstructive hydrocephalus. Figure is available in color online only.

and pseudomeningocele has been described after posterior fossa decompression for Chiari malformation. ${ }^{6,11,12}$ In these cases, hydrocephalus resolution occurred after temporary ventricular drain placement or permanent shunting. ${ }^{6,11,12}$ The subdural fluid collections and the hydrocephalus in our patient were thought to be related to the pseudomeningocele, leading to a dysfunction of CSF flow. Accordingly, we decided to treat the pseudomeningocele. Closure of the dural-arachnoid defect with resultant elimination of the pseudomeningocele was the only surgical maneuver performed during reoperation and allowed for normalization of CSF flow, with disappearance of subdural fluid collections and hydrocephalus. In this report we provide unprecedented evidence that the development of a postoperative pseudomeningocele at the cervical level can be sufficient to deeply alter the CSF flow dynamics at the cranial level, with resultant hydrocephalus.

\section{Conclusions}

Hydrocephalus can occur after surgical treatment of intradural cervical tumors. In our patient, the occurrence of a postoperative pseudomeningocele led to the development of subdural fluid collections and eventually to obstructive hydrocephalus. When pseudomeningocele and hydrocephalus are present, surgical correction of the pseudomeningocele determines resolution of hydrocephalus. A proper understanding of the pathophysiological cascade giving rise to both the symptoms and the hydrocephalus allows for effective treatment of this unusual condition.

\section{Acknowledgments}

We thank Juliet Strachan for the English revision.

\section{References}

1. Andersson H, Elfverson J, Svendsen P: External hydrocephalus in infants. Childs Brain 11:398-402, 1984

2. Bahl A, Murphy M, Thomas N, Gullan R: Management of infratentorial subdural hygroma complicating foramen magnum decompression: a report of three cases. Acta Neurochir (Wien) 153:1123-1128, 2011

3. Bland LI, McDonald JV: Hydrocephalus following spinal cord schwannoma resection. Arch Neurol 49:882-885, 1992

4. Cardoso ER, Schubert R: External hydrocephalus in adults. Report of three cases. J Neurosurg 85:1143-1147, 1996

5. Cohen AR, Wisoff JH, Allen JC, Epstein F: Malignant astrocytomas of the spinal cord. J Neurosurg 70:50-54, 1989

6. Elton S, Tubbs RS, Wellons JC III, Blount JP, Grabb PA, Oakes WJ: Acute hydrocephalus following a Chiari I decompression. Pediatr Neurosurg 36:101-104, 2002

7. Huh PW, Yoo DS, Cho KS, Park CK, Kang SG, Park YS, et al: Diagnostic method for differentiating external hydrocephalus from simple subdural hygroma. J Neurosurg 105:65-70, 2006

8. Marshman LAG, Benjamin JC, Chawda SJ, David KM: Acute obstructive hydrocephalus associated with infratentorial subdural hygromas complicating Chiari malformation Type I decompression. Report of two cases and literature review. J Neurosurg 103:752-755, 2005

9. Mirone G, Cinalli G, Spennato P, Ruggiero C, Aliberti F: Hydrocephalus and spinal cord tumors: a review. Childs Nerv Syst 27:1741-1749, 2011

10. Montemurro N, Cocciaro A, Meola A, Lutzemberger L, Vannozzi R: Hydrocephalus following bilateral dumbbell-shaped C2 spinal neurofibromas resection and postoperative cervical pseudomeningocele in a patient with neurofibromatosis Type 1: a case report. Evid Based Spine Care J 5:136-138, 2014

11. Perrini P, Di Lorenzo N: Subdural fluid collections after decompression for Chiari malformation. J Neurosurg 114:1488-1490, 2011

12. Perrini P, Rawlinson A, Cowie RA, King AT: Acute external hydrocephalus complicating craniocervical decompression for syringomyelia-Chiari I complex: case report and review of the literature. Neurosurg Rev 31:331-335, 2008

13. Ranjan A, Cast IP: Symptomatic subdural hygroma as a complication of foramen magnum decompression for hindbrain herniation (Arnold-Chiari deformity). Br J Neurosurg 10:301-303, 1996

14. Rifkinson-Mann S, Wisoff JH, Epstein F: The association of hydrocephalus with intramedullary spinal cord tumors: a series of 25 patients. Neurosurgery 27:749-754, 1990

15. Yoshimoto Y, Wakai S, Hamano M: External hydrocephalus after aneurysm surgery: paradoxical response to ventricular shunting. J Neurosurg 88:485-489, 1998

\section{Disclosures}

The authors report no conflict of interest concerning the materials or methods used in this study or the findings specified in this paper.

\section{Author Contributions}

Conception and design: Benedetto, Cagnazzo. Acquisition of data: Cagnazzo, Gambacciani. Drafting the article: all authors. Critically revising the article: Perrini, Benedetto. Reviewed submitted version of manuscript: all authors.

\section{Correspondence}

Paolo Perrini, Neurosurgical Department, Azienda Ospedaliero Universitaria Pisana, Via Paradisa 2, Pisa 56127, Italy. email: p.perrini@ao-pisa.toscana.it. 